\title{
Mobile Learning Engine Moodle adaptado aos diferentes Estilos Cognitivos utilizando Hipermídia Adaptativa
}

\author{
Patricia Mariotto Mozzaquatro - Universidade de Cruz Alta /RS - \\ patriciamozzaquatro@gmail.com \\ Roseclea Duarte Medina - Universidade Federal de Santa Maria/RS - \\ roseclea.medina@gmail.com
}

\section{Resumo}

Este artigo apresenta a adaptação do ambiente virtual de aprendizagem Mobile Learning Engine Moodle aos diferentes estilos cognitivos utilizando a hipermídia adaptativa. A validação do sistema foi realizada em duas turmas onde foi ministrado o Curso sobre Softwares Educativos. Os materiais e atividades foram apresentados de acordo com os quatro Estilos cognitivos que mais se destacaram: Holista, Serialista, Divergente e Reflexivo. Os resultados inferidos mostraram que o AVA adaptado influenciou positivamente no processo de aprendizagem dos alunos e consequentemente na sua estrutura cognitiva.

Palavras - Chaves: estilos cognitivos, MLE Moodle, hipermídia adaptativa, SEDECA

\section{Mobile Learning Engine Moodle adapted to different Cognitive styles utilizing Adaptive Hipermedia}

\begin{abstract}
This paper presents the adaptation of the virtual learning environment Mobile Learning Engine Moodle to different cognitive styles by using the adaptive hypermedia. The system validation was performed in two classes where he taught the course on Educational Software. The materials and activities were presented in accordance with the four cognitive styles that stood out: holist, serialist, Divergent and Reflective. The results showed that the inferred adapted AVA has positively influenced the process of learning and consequently in their cognitive structure.
\end{abstract}

Keywords: cognitive styles, MLE Moodle, adaptive hipermedi, SEDECA

\section{Introdução}

Nas últimas décadas, segundo Almeida (2008), “a educação a distância (EAD) tomou um novo impulso que favoreceu a disseminação e a democratização do acesso à educação em diferentes formas de interação e aprendizagens”. Nesse sentido, a utilização das tecnologias de informações e comunicação (TICs) constituem um dos eixos orientadores do desenvolvimento da educação e formação, podendo ser considerada como um dos paradigmas de inovação e evolução das sociedades. No entanto, mais importante do que qualquer tecnologia é a forma como as pessoas a utilizam no seu desenvolvimento individual ou coletivo, ou seja, como as pessoas tornam as tecnologias úteis às suas vidas, tendo em conta os seus estilos cognitivos.

De acordo com as autoras, Falkembach e Tarouco (2002), "a principal meta na área educacional é a construção do conhecimento”. Pesquisas são feitas com o intuito de aprimorar estratégias de ensino para a obtenção de melhores resultados na 
aprendizagem. No atual contexto pretende-se que o aluno seja o centro de sua própria aprendizagem, em que sua experiência e interesses são peças fundamentais, identificar e saber tirar proveito do conhecimento dos estilos cognitivos, pode constituir um ótimo princípio para garantir o sucesso acadêmico dos alunos. Witkin e Goodenough (1981) definem "estilos cognitivos como formas sutis e relativamente estáveis usadas pelo sujeito para perceber, resolver problemas e aprender a se relacionar com os outros”. Ao tratar o tema estilos cognitivos surgem algumas questões, tais como: Quais são os estilos cognitivos em que se pretende refletir? Como se pode identificar os estilos cognitivos predominantes de cada pessoa? Quais são os níveis de preferência admitidos para cada estilo cognitivo?

Os estudos de (Barbosa 2007), mostram o desenvolvimento de ambientes virtuais de aprendizagem (AVAs) que suportam essa modalidade de educação, o foco será o aluno-aprendiz, em suas necessidades, objetivos, estilo cognitivo e ritmo de aprendizagem, com a finalidade de facilitar o processo de aprendizagem e gerar conhecimento. No contexto das interfaces de aprendizagem, um dos problemas comuns nos AVAs é que a maioria se limita a uma rede de páginas de hiperdocumentos estáticos e que não aproveitam todas as funcionalidades e características das aplicações hipermídia (Gasparini 2002), outro problema é que os estudantes são tratados como se tivessem sempre o mesmo perfil, conhecimento e estilo de aprendizagem. É neste contexto de variáveis que se procurou adaptar o AVA MLE Moodle (módulo extensivo do AVA Moodle acessado via dispositivo móvel) aos diferentes estilos cognitivos, com o auxílio do SEDECA, favorecendo situações de aprendizagem individualizadas.

\section{Mobile Learning (m-learning)}

O avanço das TICs têm estimulado o desenvolvimento das mais diversas e inovadoras formas de difundir conhecimento através da WWW. Neste contexto, discutese hoje o conceito de Aprendizagem com Mobilidade - Mobile Learning ou $M$ Learning definida como: processos de aprendizagem que ocorrem, necessariamente apoiados pelo uso de tecnologias de informação móveis (TIMS) e que tem como característica fundamental a mobilidade de atores humanos (Bowker 2000). O MLearning é a fusão de diversas tecnologias de processamento e comunicação de dados que permite a estudantes e professores uma maior interação. Nesse sentido, tornou-se possível o surgimento de novos ambientes de programação focados neste tipo de equipamento, onde é possível desenvolver aplicações quase que totalmente independentes de dispositivo e fabricante. A seguir é apresentado o AVA móvel Mle Moodle.

Mobile Learning Engine Moodle (Mle Moodle): é um sistema criado para dispositivos móveis, totalmente gratuito, de código aberto e personalizável, vinculado ao AVA Moodle. As especificações podem ser adaptadas conforme necessário com WML, PHP e MySQL. O ambiente fornece a interface para o dispositivo móvel, quaisquer alterações efetuadas ao Moodle são automaticamente convertidos para os dispositivos Mle Moodle. O MLE Moodle integra os seguintes recursos que são acessados via dispositivo móvel e que estão presente no E-Learning Moodle: Lição, Quiz, Recursos, Fórum, Questionário, Wiki, Banco de Dados e Sistema de mensagens instantâneas. Apresenta também recursos parcialmente específicos para m-learning: Flashcard Trainer, Mobile Learning Objects, Mobile tags. 


\section{A importância de diagnosticar estilos cognitivos para o ensino a distância}

A EAD contempla uma comunidade virtual de aprendizagem onde aluno e professor passam a ser parceiros. Essas relações ocorrem em um contexto cultural dinâmico e em constante evolução e, podem, no processo educacional promover a aprendizagem através da troca de informações e conhecimentos entre os principais envolvidos: professores e alunos. Um aspecto de grande relevância na EAD são os estilos cognitivos dos integrantes do processo educacional. Cabe salientar que esses estilos influenciam aspectos como: atitudes, valores, interação social, resolução de problemas, entre outros.

Segundo Geller (2004), "o conhecimento do estilo cognitivo do aluno é importante para o professor que almeja a aprendizagem desse sujeito". Esse conhecimento também é de fundamental importância para apoiar a adaptação de um AVA que mediará o processo de educação não presencial, uma vez que os estilos cognitivos predominantes podem influenciar o modo como os alunos aprendem, como os professores ensinam e como juntos eles interagem. Kulski e Quinton (2002), afirmam que: "Há uma preocupação crescente nos investigadores com a construção de AVAs, de modo que sejam adaptáveis aos diferentes estilos cognitivos, ou seja, na concepção e estruturação dos conteúdos, numa perspectiva da sua reutilização em novos ambientes de educação”. As potencialidades das tecnologias de informação e da comunicação, mais precisamente as características multimídia, podem contribuir para a construção de materiais adaptados ao estilo particular de cada aluno, sem a preocupação de uma esteriotipagem ou categorização dos alunos (Gordon e Bull 2004). Hodgins (2000), afirma que "a personalização de experiências de aprendizagem exige conhecer o aluno". O mesmo autor, acrescenta que quanto mais se conhecer o aluno para a construção do sistema de aprendizagem, maior é a oportunidade para lhe proporcionar informação adequada. A chave para o sucesso da aprendizagem em AVAs é reconhecer que as diferenças de aprendizagem existem e que as mesmas devem ser reconhecidas, salientando que uma mesma abordagem não funciona do mesmo modo para todos os alunos (Palloff e Pratt 2003).

\subsection{Estilos Cognitivos}

Na literatura existe diversas conceituações para estilos cognitivos (Geller 2004). Nesta pesquisa, entende-se que os estilos cognitivos referem-se ao meio preferido pelo qual um indivíduo processa a informação, descrevendo o seu modo típico de pensar, relembrar ou resolver problemas. Em síntese, os estilos cognitivos poderiam ser utilizados para auxiliar na elaboração das estratégias instrucionais mais eficazes para um dado indivíduo. Quando se conhece os diferentes estilos cognitivos dos alunos e o ato de ensinar é adaptado a esse fato, os alunos podem tornar-se mais responsáveis e atingem, de modo significativo, níveis mais altos de aprendizagem (Bender 2003). A identificação dos estilos cognitivos é importante no sentido de incitar uma ligação entre o ensino e os modos como os alunos preferem aprender e, se assim for, os alunos demonstram melhores resultados e um desejo mais forte de aprender (Given 2002). A investigação associada aos estilos cognitivos refere que os alunos aprendam melhor quando a abordagem ao conhecimento é adequada aos seus modos preferidos de aprender. Quanto às dimensões dos estilos cognitivos, um grande número delas encontra-se disponível na literatura, as quais são descritas na próxima subseção. 


\subsubsection{As dimensões dos estilos cognitivos}

Os autores Felder e Soloman formularam um modelo de estilos cognitivos com dimensões relevantes para a educação científica. Os autores definiram estilos cognitivos como preferências e características dominantes no modo como as pessoas recebem e processam as informações (Felder e Soloman 1993). O modelo proposto define quatro dimensões de estilos: Retenção da informação (Visual-Verbal), Percepção da informação (Sensorial-Intuitivo), Processamento da informação (Ativo-Reflexivo) e Organização da informação (Seqüencial-Global). Para a identificação dos estilos cognitivos foi desenvolvido um instrumento, denominado Index of Learning Styles

Questionnaire (ILS) disponível em: http://www.engr.ncsu.edu/learning styles/ilsweb.html . O modelo proposto por Honey e Munford considera que as principais características das pessoas podem ser integradas em quatro estilos cognitivos: Ativo, Reflexivo, Teórico e Pragmático. Admitem ainda, que em cada pessoa é possível identificar características dos vários estilos cognitivos, embora geralmente, cada pessoa possua um estilo dominante (Honey e Munford 2000). Foi criado o Cuestionario HoneyAlonso de Estilos de Aprendizaje disponível em: http://www.estilosdeapren dizaje.es/chaea/chaea.htm, na versão Portuguesa, com o objetivo de ajudar os alunos a estarem atentos aos seus pontos fortes e fracos dos estilos cognitivos. Para Bariani, os estilos cognitivos são formas relativamente estáveis referentes às características da estrutura cognitiva de uma pessoa, que são definidas, em parte, por fatores biológicos, sendo influenciadas pela cultura e modificadas a partir da influência direta ou indireta de novos eventos (Bariani 1998). A autora utiliza quatro dimensões: Dependência de campo - Independência de campo, Reflexividade de resposta - Impulsividade, Convergência de pensamento - Divergência de pensamento e Holista - Serialista. Para identificar estilos cognitivos foi desenvolvido um instrumento de coleta de dados, composto por modelos de questionário para obtenção de dados pessoais e outro do tipo escalar - uma escala do tipo Likert. (Bariani 1998).

\section{Hipermídia adaptativa}

O autor Brusilovsky (1998) define Hipermídia Adaptativa como: "Todo sistema de hipertexto e/ou hipermídia que reflita algumas características, de seus diferentes usuários, em modelos e aplique, esses modelos, na adaptação de diversos aspectos visíveis do sistema às necessidades, desejos e preferências de cada usuário”. O objetivo da Hipermídia Adaptativa é oferecer aos usuários interfaces cujo estilo, conteúdo, recursos e links sejam automaticamente selecionados, reunidos e apresentados de acordo com seus objetivos, necessidades e preferências (Falkembach e Tarouco 2002). Além do conhecimento dos aspectos que devem ser levados em conta na adaptação, também é necessário saber quais as características do sistema que podem ser adaptadas para diferentes usuários, são apresentadas: adaptação em nível de conteúdo e adaptação em nível de links. A adaptação em nível de conteúdo é realizada através de técnicas de apresentação adaptativa, enquanto que a adaptação em nível de links é realizada através de técnicas de suporte adaptativo à navegação. A seguir são apresentados métodos e técnicas de apresentação adaptativa e navegação adaptativa. Segundo Palazzo as técnicas de apresentação adaptativa são: Texto Condicional, Strechtext, Fragmentos Variantes, Páginas Variantes e Frames (Palazzo 2000). Os métodos para a adaptação, segundo o mesmo autor são: Explicação Adicional, Explicação Requerida, Explicação Comparativa, Explicação Variante e Classificação de Fragmentos (Palazzo 2000). A 
adaptação na navegação introduz os componentes da interface passo a passo, e por Palazzo (2000), as técnicas para modelar uma navegação adaptativa são: Orientação Direta, Classificação Adaptativa, Ocultação, Anotação Adaptativa e Mapas Adaptativos. Os métodos que dão suporte à navegação adaptativa são: Condução Global, Condução Local, Suporte à Orientação Local e Orientação Global.

\section{Metodologia}

A pesquisa consistiu na adaptação do AVA móvel MLE Moodle aos diferentes estilos cognitivos utilizando técnicas de hipermídia adaptativa. Foi criado e aplicado um Sistema Especialista para diagnosticar estilos cognitivos, SEDECA. O estilo de aprendizagem foi identificado através do Instrumento "Questionário" embasado nos instrumentos de investigação propostos por Felder - Soloman (1993), Honey - Munford (2000) e Bariani (1998). O resultado do teste indicou as preferências nos estilos: Sensorial, Intuitivo,Visual, Verbal, Ativo, Reflexivo, Seqüencial, Global, Teórico, Pragmático, Impulsivo, Convergente, Divergente, Holista, Serialista, Independência de Campo e Dependência de Campo. O sistema inicialmente foi constituído por 68 questões objetivas (4 questões de cada estilo) em que o aluno deveria escolher uma das quatro alternativas apresentadas (C), (CT), (D), (DT). A partir dos dados coletados no SEDECA, foi possível identificar os quatro estilos cognitivos que mais se destacaram, como também as ferramentas de comunicação preferenciais a cada estilo, a fim de subsidiar o processo de modelagem e implementação do AVA a ser adaptado. O questionário foi aplicado aos alunos dos cursos de Graduação e Pós- Graduação da modalidade EAD. Constatou-se que os quatro estilos predominantes foram Divergente (28\%), Holista (26\%), Reflexivo (24\%) e Serialista (22\%). Em relação as ferramentas de comunicação preferenciais aos estilos predominantes destacaram-se a mensagem e o fórum (estilo Divergente e Reflexivo). Estilo Holista (chat e mensagem) e o estilo Serialista (chat e fórum). Prosseguindo, foi aplicado um Questionário On-line complementar ao SEDECA. O questionário foi composto por 13 questões, sendo elas objetivas e dissertativas. O conteúdo abordado no questionário referiu-se a indicação das ferramentas de comunicação e formato do material. Após estabelecer -se os indicadores para a adaptação, iniciou-se o processo de modelagem. A modelagem foi realizada através de diagramas UML. A Figura 1 apresenta o diagrama de Casos de uso

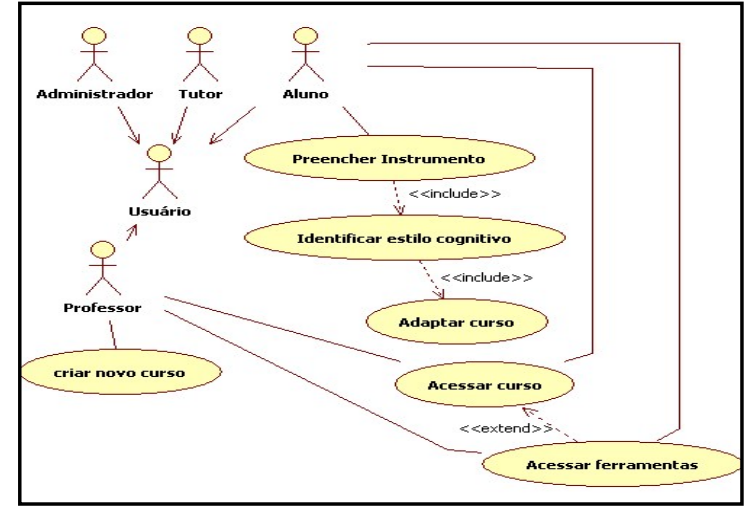

Figura 1- Diagrama de Caso de Uso Geral

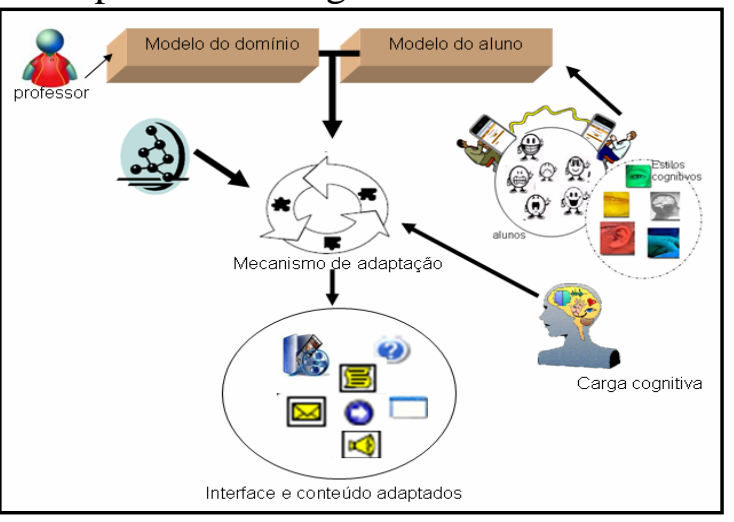

Figura 2- Arquitetura proposta

Na Figura 2 é apresentada a arquitetura básica para adaptação do AVA Mle Moodle ao estilo cognitivo do aluno. A arquitetura apresenta três componentes básicos: Modelo do aluno que descreve o usuário para o sistema, nele é representado suas preferências de aprendizagem, conhecimentos e estilos cognitivos. Este modelo contém uma representação do estilo cognitivo do aluno desde o momento em que interage com 
o sistema (responde o questionário o qual irá detectar seu estilo cognitivo predominante); Modelo de domínio: este modelo é conhecido como base de conhecimento do domínio, onde é representado o material instrucional adaptado ao estilo cognitivo do aluno, que posteriormente será utilizado pelo mesmo; Mecanismo de Adaptação: utiliza o modelo do aluno e o modelo de domínio para prover, de forma dinâmica a adaptação do AVA móvel ao estilo cognitivo do aluno. Na adaptação proposta foi utilizada técnica (Página Variante) e método (Explicação variante) de hipermídia adaptativa. O material instrucional e as ferramentas de interação síncronas e assíncronas foram apresentadas aos alunos conforme seu estilo cognitivo.

\section{Mobile Learning Engine Moodle adaptado}

O trabalho proposto buscou adaptar um AVA móvel aos diferentes estilos cognitivos. A adaptação foi realizada no AVA móvel Mle Moodle. A adaptação do conteúdo baseou-se em estereótipos, classificando os alunos de acordo com os estilos cognitivos. Um aluno pode estar compreendido entre quatro estilos cognitivos: Reflexivo, Serialista, Holista e Divergente. Para cada um destes estilos, foram definidas as melhores formas de apresentação de conteúdo, baseadas nos trabalhos de Bariani (1998) e Geller (2004). Através desse modelo, foram estabelecidas 14 técnicas de adaptação, sendo 9 de conteúdo e 5 avaliação, e definidas quais as mais adequadas para cada estilo cognitivo. As técnicas utilizadas são: No estilo Holista o material a ser apresentado ao aluno encontra-se nas categorias: Texto (Links, Artigos, Livros); Imagem (Diagramas ou Mapas); Atividades (Pesquisa, Resenha crítica); Ferramentas (Chat, Mensagem). O Estilo Serialista sugere o Texto no formato de (Tutoriais e materiais apresentados na forma de tópicos); Imagens (Vídeo e Esquemas); Atividades (Fórum, Questionário e Pesquisa); Ferramentas (Chat e Fórum). No Estilo Divergente é apresentado: Texto ( Links e formato Tópicos); Imagens (Vídeo, Diagramas ou Mapas); Atividades (Fórum, Mapa conceitual, Pesquisa e Questionário); Ferramentas (Fórum e Mensagem). O Estilo Reflexivo apresenta: Texto (Capítulo de Livros, Artigo, Tutorial); Imagens (Vídeo, Diagramas ou Mapas); Atividades (Fórum, Resenha Crítica, Questionário, Pesquisa); Ferramentas (Fórum, Mensagem). Agregado ao ambiente, existe um questionário que deverá obrigatoriamente ser preenchido por todos os alunos ao iniciarem um curso. Esse questionário tem como função identificar o estilo cognitivo predominante. Para o aluno ser individualizado no sistema, deve efetuar um login inicial, desta forma suas ações serão registradas e acompanhadas. Após será mostrada uma página contendo um instrumento (Figura 3), através do qual será estabelecido o estilo cognitivo dominante, que será armazenado no banco de dados e comporá o Modulo Adaptado. A figura 4 apresenta o resultado do questionário.

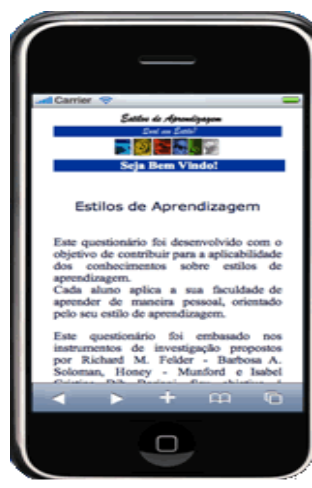

Figura 3- Questionário

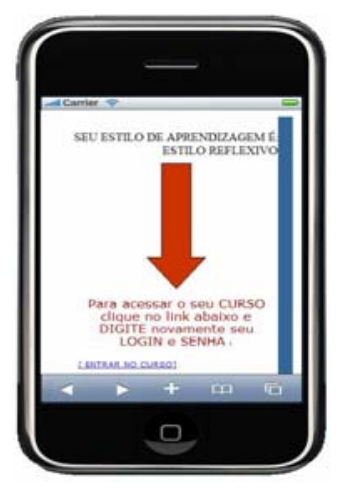

Figura 4- Resultado

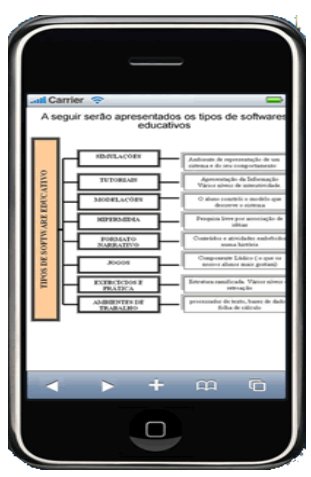

Figura 5- Holista

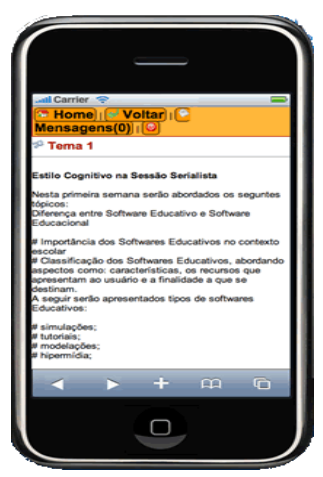

Figura 6- Serialista 
A identificação do estilo de aprendizagem servirá para indicar quais as ferramentas e materiais preferenciais serão mais adequados a seus estilos cognitivos. Somente depois de respondido o questionário, o aluno terá acesso à tela inicial do sistema. A adaptação do conteúdo apresentada no Curso Softwares Educativos, segundo dois estilos cognitivos diferentes é demonstrada nas figuras 5 e 6 respectivamente, 0 conteúdo foi disponibilizado a alunos que possuem os estilos cognitivos Holista e Serialista. A Figura 5, cujo estilo predominante é Holista apresentando texto e diagrama demonstra a implementação da técnica de Página Variante (uma página pode apresentar vários conceitos onde cada um deles é formado por fragmentos variantes que são combinados de acordo com o estilo de aprendizagem do aluno). A figura 6 apresenta o mesmo conteúdo sob a visão de um aluno com estilo cognitivo dominante Serialista, que visualiza as informações sob a forma de tópicos.

\section{Resultados e Discussão}

O AVA móvel adaptado foi validado por 25 alunos dos Cursos de Graduação e Pós Graduação a Distância do Sistema Universidade Aberta do Brasil (UAB). Foi ministrado um Curso sobre Softwares Educativos integrando uma população de 50 alunos, sendo a Turma A composta por 25 alunos acessando o ambiente adaptado e a Turma B também com 25 alunos acessando ambiente não adaptado. O acesso aos ambientes foi realizado via desktop e dispositivo móvel. O material integrante do Curso foi apresentado de acordo com os quatro estilos cognitivos: Holista, Serialista, Divergente e Reflexivo. As atividades existentes foram diferenciadas de acordo com os estilos cognitivos, para compor a avaliação final. A constituição formal deste trabalho deu-se através de análises estatísticas e análise de conteúdo, utilizadas com o propósito de estudar e analisar se o prévio conhecimento dos estilos cognitivos irá influenciar positivamente no processo de ensino - aprendizagem. Analisando-se os resultados da validação quanto à interação com o sistema e à adaptação de conteúdo, verificou-se que todos os itens avaliados tiveram resultados positivos com relação ao Ambiente Adaptado.

Destaca-se que a 83\% dos alunos que participaram da validação, consideram válida a utilização do sistema implementado. Com relação à adaptação das atividades, $100 \%$ dos alunos consideraram que o tipo de atividade proposta para o seu estilo cognitivo favoreceu o processo de aprendizagem. Quanto aos estilos cognitivos diagnosticados, 83\% afirmam que a forma de apresentação dos conteúdos favoreceu a aprendizagem. Além dos resultados apresentados, os alunos que participaram da validação tiveram um espaço para realizar considerações sobre o AVA adaptado, entre as quais destacou-se: "A forma de apresentação do conteúdo foi bastante satisfatória. Senti muita diferença, pois consegui visualizar o conteúdo de forma prazerosa”; "O acesso ao ambiente pelo iphone foi normal, apenas encontrei algumas limitações em relação ao tamanho dos arquivos e imagens. Mas foi muito produtivo, pois em qualquer lugar e a qualquer tempo eu pude ler os materiais e fazer as atividades”. Nas questões que envolveram a interface e a navegação, todos os percentuais apresentados foram positivos com relação ao sistema implementado.

Conforme exposto anteriormente, o Curso foi ministrado para duas turmas. A partir dos dados coletados foram feitas demonstrações gráficas apresentando os resultados obtidos. A Figura 7 apresenta o total de participantes x desistentes integrantes das respectivas turmas. 


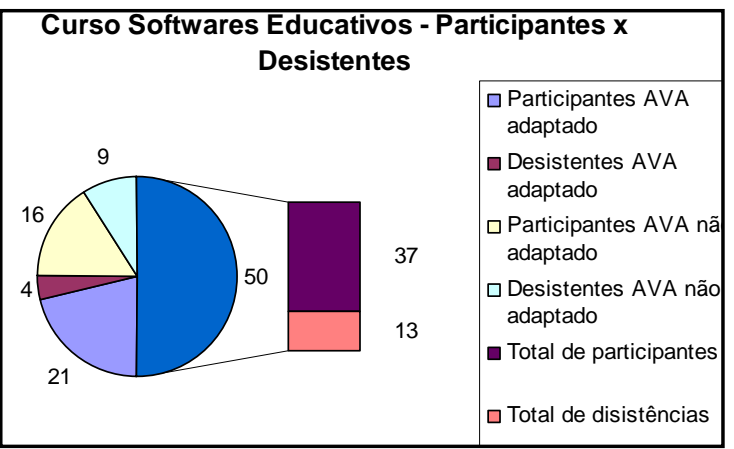

Figura 7- Participantes x Desistentes

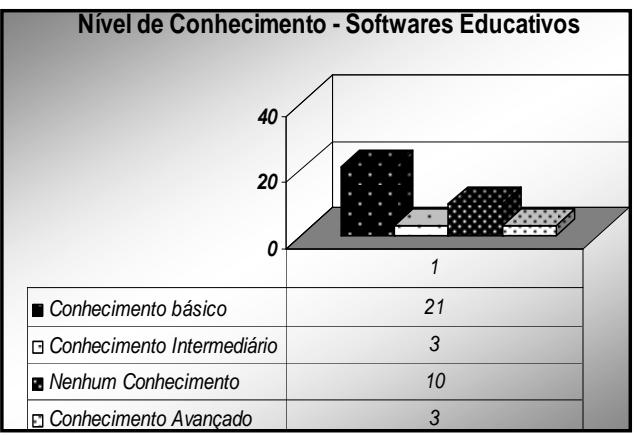

Figura 8- Nível de conhecimento

Conforme observa-se na Figura 7, a Turma A que utilizou o AVA adaptado apresentou 21 participantes com apenas 4 desistências. Em contrapartida, a Turma B que utilizou o AVA não adaptado apresentou 16 participantes com 9 desistências. Dos 21 participantes da Turma A, 6 realizaram o curso via dispositivo móvel e 15 via desktop. Ao efetuar a inscrição no Curso, os alunos responderam a um questionário. A Figura 8 apresenta o nível de conhecimentos os alunos em relação ao tema Softwares Educativos antes do início do Curso. Observa-se que a maioria dos alunos apresentou conhecimento básico (21). Quanto aos níveis de conhecimento Intermediário e Avançado (3). Em contrapartida, um número elevado de participantes apresentaram nenhum conhecimento sobre o tema (10). Através da ferramenta Relatório dos AVAs foi possível verificar a quantidade de acessos dos integrantes das Turmas A e B quanto aos materiais para leitura e atividades. A Figura 9 demonstra este processo.

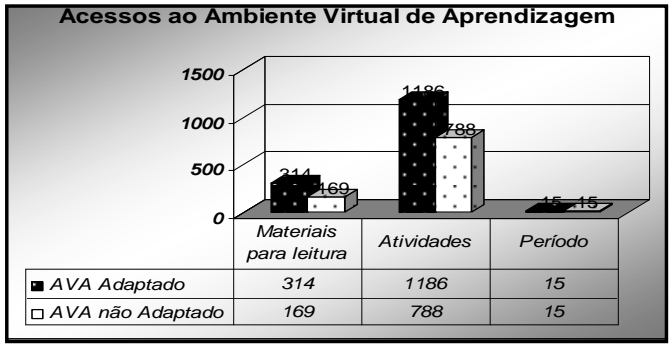

Figura 9- Acessos aos AVAs

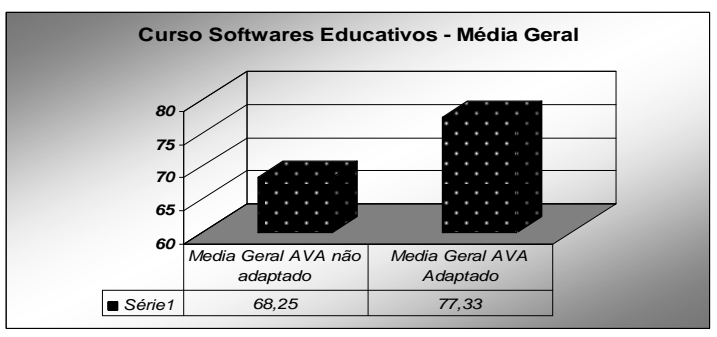

Figura 10- Média Geral

Conforme observado na Figura 9, a quantidade de acessos aos materiais para leitura e atividades foram maiores no AVA adaptado (314 acessos aos materiais e 1186 acessos às atividades). Em contrapartida, no AVA não adaptado (169 acessos aos materiais e 788 acessos às atividades). A Figura 10 apresentou a média geral dos alunos integrantes da Turma A (AVA adaptado) e Turma B (AVA não adaptado). O Curso ministrado compõe-se de seis atividades (Fórum, Resenha Crítica, Questionário, Mapa conceitual, Pesquisa e Glossário), sendo três delas correspondentes a cada semana. Observou-se que a média geral dos alunos participantes da Turma A foi 77,33\%, enquanto os alunos integrantes da Turma B apresentaram 68,25\%, constatou-se um diferencial de $9,08 \%$.

\section{Considerações Finais}

Acredita-se que o trabalho, que aqui se encerra, tenha alcançado seus objetivos, bem como contribuído para uma evolução nas pesquisas sobre o tema estilos cognitivos. 
O ambiente adaptado foi validado no Curso sobre Softwares Educativos com alunos dos Cursos de Especialização (TICs Aplicadas à Educação, Mídias na Educação, Educação Ambiental e Mestrado em Geografia) e Graduação (Pedagogia e Letras e Matemática) sistema UAB/UFSM. Conforme a análise dos resultados referentes às médias gerais, número de acessos aos AVAs, interação com o sistema e à adaptação de conteúdo considera-se que os objetivos definidos neste artigo foram alcançados na sua totalidade. As médias gerais da Turma A que utilizou o AVA adaptado ao estilo cognitivo do aluno diferenciou-se com percentual de 9,08\% maior que a Turma B (AVA não adaptado). Percebeu-se que o AVA adaptado proporcionou maior motivação dos alunos a interagirem com os materiais e atividades que compõe o ambiente. Deve-se ressaltar que a qualidade da atuação da autora na Turma A (AVA adaptado) e Turma B (AVA não adaptado) foi equivalente.

Logo, estes dados levam-nos a crer que o prévio conhecimento dos estilos cognitivos irá influenciar positivamente na estrutura cognitiva do aluno. Espera-se contribuir com novos olhares para aprimorar estratégias de ensino que proporcionem melhorias para o processo de ensino - aprendizagem, contribuindo assim, para a formação integral do acadêmico. Procurou-se, antes de tudo, entender que o processo de ensino e aprendizagem alcança seus objetivos quando professor e aluno encontram-se dispostos a experimentar novas alternativas que promovam e justifiquem a aprendizagem.

\section{Referências}

ALMEIDA, Maria Elizabeth Bianconcini de. Tecnologia e educação a distância: abordagens e contribuições dos ambientes digitais e interativos de aprendizagem, 2008. Disponível em:<http://www.anped.org.br/reunioes/26/ trabalhos /ma riaeliza bethalmeida.rtf $>$ Acesso em: 20 Dez. 2009.

BARBOSA, Débora Nice Ferrari. Um modelo de educação ubíqua orientado à consciência do contexto do aprendiz. Universidade Federal do Rio Grande do Sul. Porto Alegre, 2007.

BARIANI, I. C. Estilos Cognitivos de Universitários e Iniciação Científica. Campinas: UNICAMP. Tese (Doutorado em Educação), Faculdade de Educação, Universidade Estadual de Campinas, 1998.

BENDER, T. "Discussion - based online teaching to enhance student learning: Theory, practice and assessment”. Sterling, Virginia: Stylus Publishing, LLC, 2003.

BOWKER, R.R. "Wireless Trainning or m-learning” is here: first movers in the pool. Lifelong learning”. Market report, 2000, p. 5-22.

BRUSILOVSKI, Peter e MAYBURY, Mark. "From adaptive hypermedia to the adaptive Web“. In: Communications of the ACM. v.45, n.5, 1998, pp. 31-33.

FALKEMBACH, Gilse Antoninha Morgental; TAROUCO, Liane Rockembach. Hipermídia adaptativa: uma opção para o desenvolvimento de sistemas educacionais visando uma aprendizagem mais efetiva. Universidade Federal do Rio Grande do Sul. PGIE - Pós Graduação em Informática na Educação, 2002.

FELDER, R. M. "Reaching the Second Tier: learning and teaching styles in college science education”. Journal of College Science Teaching, 1993. v.23, n.5, p-286-290, http://www.ncsu.edu/felder-public/Papers/Secondtier.html, Julho, 2009. 
GARCÍA, Catalina M. Alonso; GIL, Domingo J. Gallego; CUÉ, José Luis García “Cuestionario Honey-Alonso de Estilos de Aprendizaje CHAEA”, 2009. Disponível em <http://www.estilosdeaprendizaje.es/chaea/chaea.htm>. Acesso em: Jul. 2009.

GASPARINI, Isabela. Concepção de Interfaces WWW Adaptativas para EAD. Cadernos de Informática. Porto Alegre, v.2, n.1, 2002, p.71-76.

GELLER, M. Educação a Distância e Estilos Cognitivos: construindo um novo olhar sobre os ambientes virtuais. Porto Alegre: UFRGS. Tese (Doutorado em Informática na Educação), Programa de Pós-Graduação em Informática na Educação, Universidade Federal do Rio Grande do Sul, 2004.

GIVEN, B. K. "The overlap between brain research and research on learning style”, In S. J. Armstrong et al. (Eds.), Learning Styles: Realibility \& Validity, Proceedings of the 7 th Annual ELSIN Conference. 173-178. Ghent: Ghent University. Belgium \& ELSIN, 2002.

GORDON, D e BULL, G. "The Nexus explored: A generalised model of learning styles”, In R. Ferdig e C. Crawford e R. Carisen e N. Davis e J, Price e R. Weber e D. A. Willis (Eds.), Information Technology \& Teacher Education Annual: Proceedings of SITE 2004, pp. 917-925. Norfolk, VA: Association for the Advancement of Computing in Education.

HODGINS, H. W. "The future of learning objects”, 2000. Disponível em $<$ http://www.reusability.org/read/chepters/hodgins.doc> . Acesso em: Mar. 2010.

HONEY, P. e MUMFORD. A. “The Learning Styles helper's guide”. Maldenhead Berks: Peter Honey Publications, 2000.

KULSKI, M; QUINTON, S. "Personalising the online learning experience", In S. J. Armstrong et al. (Eds.), Learning Styles: Realibility \& Validity, Proceedings of the 7 th Annual ELSIN Conference. 221-225. Ghent: Ghent University. Belgium \& ELSIN, 2002.

PALAZZO, Luiz Antônio Moro. Modelos Pró-ativos para Hipermídia Adaptativa. Tese de Doutorado. Porto Alegre: PPGC-UFRGS, 2000.

PALLOFF, R; PRATT, K. "The virtual student: A profile and guide to working with online learners”. San Francisco: Jossey- Bass Publishers, 2003.

SOLOMAN, Barbara A. e FELDER, Richard M.. "Index of Learning Styles Questionnaire”. Department of Chemical Engineering North Carolina State University Raleigh, NC 27695-7905, 1993. Disponível em $<$ http://www.engr.ncsu.e du/learningstyles/ilswe b.html>. Acesso em: Ago. 2009.

WITKIN, H. A. e GOODENOUGH, D. R. "Cognitive Style: essence and origins". New York: International Universities Press, 1981 